\title{
Sonoluminescence light emission
}

\author{
Sascha Hilgenfeldt \\ Division of Engineering and Applied Sciences, Harvard University, 29 Oxford St, \\ Cambridge, Massachusetts 02138 \\ Siegfried Grossmann \\ Fachbereich Physik der Universität Marburg, Renthof 6, D-35032 Marburg, Germany \\ Detlef Lohse \\ Department of Applied Physics and J. M. Burgers Centre for Fluid Dynamics, University of Twente, \\ P.O. Box 217, 7500 AE Enschede, The Netherlands
}

(Received 22 July 1998; accepted 28 December 1998)

\begin{abstract}
Single bubble sonoluminescence is not an exotic phenomenon but can quantitatively be accounted for by applying a few well-known, simple concepts: the Rayleigh-Plesset dynamics of the bubble's radius, polytropic uniform heating of the gas inside the bubble during collapse, the dissociation of molecular gases, and thermal radiation of the remaining hot noble gas, where its finite opacity (transparency for its own radiation) is essential. A system of equations based on these ingredients correctly describes the widths, shapes, intensities, and spectra of the emitted light pulses, all as a function of the experimentally adjustable parameters, namely, driving pressure, driving frequency, water temperature, and the concentration and type of the dissolved gas. The theory predicts that the pulse width of strongly forced xenon bubbles should show a wavelength dependence, in contrast to argon bubbles. () 1999 American Institute of Physics. [S1070-6631(99)01704-3]
\end{abstract}

\section{INTRODUCTION}

\section{A. Converting sound into light}

Single bubble sonoluminescence (SBSL) is a phenomenon that was discovered not even a decade ago, ${ }^{1}$ and has triggered a large number of experimental, numerical, and theoretical publications in subsequent years. At first, the process of converting sound energy into visible light seemed mysterious in almost all of its aspects. The light is emitted in the collapse phase of the oscillation cycle of a $\mu \mathrm{m}$-sized gas bubble driven by a standing ultrasound wave excited in a glass flask (cf. Refs. 1, 2 for the experimental setup). In a small parameter range of driving pressure amplitudes $P_{a}$ (typically $1.2-1.5 \mathrm{~atm}$ ) and gas concentrations in the liquid $c_{\infty}$ (e.g., $\approx 0.2 \%-0.4 \%$ of the saturation concentration $c_{0}$ for argon), the light pulses are emitted very regularly, once per cycle. Despite the violent collapses, the bubble remains stable for many hours.

Two basic questions arise: (i) What is the mechanism of the light emission and (ii) what determines the parameter regime of SBSL?

(i) Light emission: A large number of different models have been suggested to explain the mechanism of light radiation, ranging from bremsstrahlung, ${ }^{3,4}$ collision-induced emission $^{5}$ and emission from electrons in voids ${ }^{6}$ to fractoluminescence. ${ }^{7}$ Many of these approaches require a detailed description of the gasdynamics inside the bubble to include, e.g., the effects of thermal conduction ${ }^{8-10}$ or the possible creation of inward-focusing shock waves. ${ }^{3,4,11}$ The most elaborate model today is probably the one presented by Moss et al. ${ }^{4}$ including full PDE numerics for the bubble interior, computing temperature profiles and shock wave propagation inside the bubble. These calculations arrive at realistic numbers for emission intensities and pulse widths ${ }^{4}$ by taking into account opacity (i.e., the degree of photon absorption in the medium) and plasma physical processes such as the cooling of the gas by plasma heat conduction. Apart from presenting a formidable task concerning the necessary numerical effort, detailed calculations have also suffered from modeling uncertainties, because very little reliable data is available about the microscopic and macroscopic physical properties of a gas at the temperatures and pressures encountered inside a SL bubble.

(ii) Parameter regime of SBSL: The basic experimental parameters that can be varied are the forcing pressure amplitude, the forcing frequency, the water temperature, the gas concentration, and the gas type. The hydrodynamical/ chemical approach to SBSL, ${ }^{12-19}$ which for self-consistency is briefly outlined in the following section, provides a quantitative explanation for the parameter dependences of SBSL. Moreover, the theory yields a number of predictions (see also Ref. 20), many of which have been confirmed meanwhile in experiment. ${ }^{2,21-24}$

The motivation for the present work is the question of whether the hydrodynamical/chemical approach can be extended to compute light emission, maintaining its simple character. Indeed, this turns out to be possible. The central idea is to include thermal radiation in the model, taking special care to consider the finite opacity of the heated gas, following Moss et al. ${ }^{4}$ and succeeding papers. ${ }^{16,25,26}$

\section{B. The merits of the bubble dynamical approach}

A surprisingly accurate description of the radial oscillations of a bubble (even for very nonlinear oscillations) has 
been known for a long time in form of the Rayleigh-Plesset (RP) equation, a nonlinear ODE for the bubble radius $R$, whose interior is taken to be uniform: ${ }^{27-30}$

$$
\begin{aligned}
\rho_{l}\left(R \ddot{R}+\frac{3}{2} \dot{R}^{2}\right)= & p_{\mathrm{gas}}[R(t)]+p_{\mathrm{vap}}-P(t)-P_{0} \\
& +\frac{R}{c_{l}} \frac{d}{d t} p_{\mathrm{gas}}[R(t)]-4 \eta_{l} \frac{\dot{R}}{R}-\frac{2 \sigma}{R} .
\end{aligned}
$$

Here, $p_{\text {gas }}$ is the gas pressure inside the bubble, modeled by a van der Waals-type process equation,

$$
\dot{p}_{\text {gas }}(R, t)=\frac{d}{d t} p_{\text {gas }}[R(t)]=-\gamma(R, \dot{R}, T) \frac{3 R^{2} \dot{R}}{R^{3}-h^{3}} p_{\text {gas }},
$$

where $h$ represents the (collective) van der Waals hard core radius. The effective polytropic exponent $\gamma$ describes the degree of isothermality or adiabaticity of the bubble motion at a given time. Here $\gamma$ is, in general, a function of $R, \dot{R}$, and the gas temperature $T$, which will be discussed in detail below. If $\gamma$ can be assumed constant throughout the oscillation, (2) can be integrated to yield

$$
p_{\text {gas }}[R(t)]=\left(P_{0}+\frac{2 \sigma}{R_{0}}\right)\left(\frac{R_{0}^{3}-h^{3}}{R^{3}(t)-h^{3}}\right)^{\gamma},
$$

$R_{0}$ being the ambient bubble radius (stationary radius under normal temperature and pressure). Here $R_{0}$ is related to $h$ via $h=R_{0} / 8.86$ (for argon). ${ }^{31}$ The physical parameters in (1)(3) are the liquid density $\rho_{l}$, viscosity $\eta_{l}$, and speed of sound $c_{l}$, the surface tension $\sigma$, the vapor pressure $p_{\text {vap }}$ of water, and the constant ambient pressure $P_{0}=1 \mathrm{~atm}$. We will take the material constants to have the values for the case of argon gas dissolved in water at $20^{\circ} \mathrm{C}$ throughout this study, unless otherwise noted. Here $P(t)=-P_{a} \cos \omega t$ is the external ultrasound driving, taken to be a spatially homogeneous standing wave. The driving frequency $f=\omega / 2 \pi$ is typically $20-40 \mathrm{kHz}$ in today's experiments. Vapor pressure is crudely represented by the term $p_{\text {vap }}$, whose value is bounded from below by the vapor pressure of water at the ambient temperature, thus setting a lower limit to the bubble's interior pressure at expansion. Note that (1) contains viscous damping and sound damping (the term $\propto 1 / c_{l}$ ), but no explicit thermal damping term like the one calculated for linear oscillations by Prosperetti in Ref. 32. For argon bubbles of the size and subject to the driving frequencies in SBSL experiments, the first two damping effects are more important in this linear limit. ${ }^{33}$ The modeling of the SBSL bubble dynamics was recently refined by including thermal damping effects. ${ }^{19}$

Figure 1(a) shows an oscillation period of the typical $R(t)$ dynamics of a bubble in the SL parameter regime ( $f$ $\left.=20 \mathrm{kHz}, P_{a}=1.3 \mathrm{~atm}, R_{0}=5.0 \mu \mathrm{m}\right)$. The light pulse is emitted at the point of minimum radius after the fast collapse following the slow expansion to about ten times the ambient radius. Upon collapse, the bubble radius becomes comparable to $h$ and the density reaches values similar to those in condensed matter. The strong collapsing dynamics of such a bubble provides the extreme energy focusing necessary to
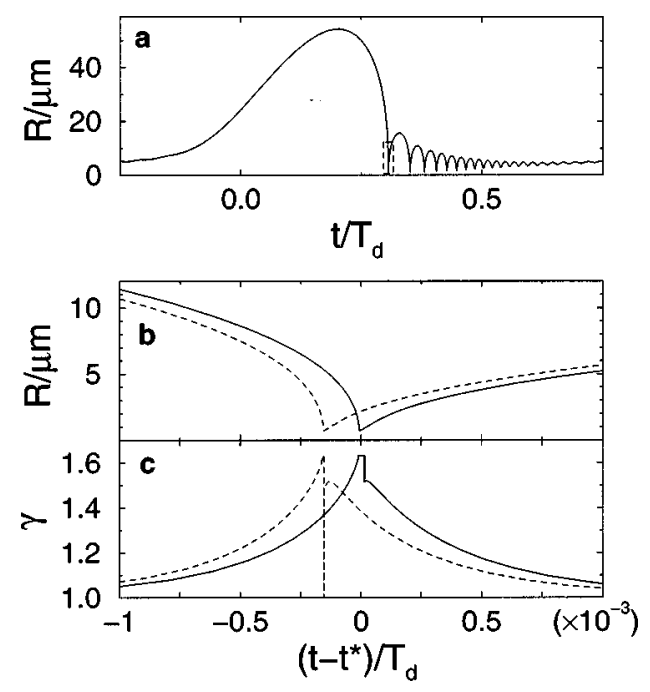

FIG. 1. (a) Time series $R(t)$ for $f=20 \mathrm{kHz}, P_{a}=1.3 \mathrm{~atm}$, and $R_{0}$ $=5.0 \mu \mathrm{m}$, computed from the complete system (1), (4), (6)-(10), (11) over one driving cycle. The time axis is normalized with the driving period $T_{d}$ $=1 / f$. The dashed box indicates the range of plots (b) and (c). (b) A close-up of $R(t)$ (solid line) around the instant $t^{*}$ of minimum radius. Note the asymmetric collapse-rebound behavior. The dashed line gives the dynamics as computed without the $\gamma(t)$ modification discussed in Sec. II E. On the scale of (a) these two graphs are indistinguishable. For the same two cases, (c) gives the variation of $\gamma$ in time. The modification only affects the innermost $\sim 10^{-5} T_{d}$ around $t^{*}$.

create light from sound. Surprisingly, for appropriate parameter values the bubble survives this catastrophic collapse and repeats the same oscillation with high precision for many millions of cycles. As mentioned above, this stable SBSL is observed in a small portion of the parameter space of driving pressure amplitude $P_{a}$ and relative gas concentrations $c_{\infty} / c_{0}$.

In the past years, the parameter restrictions on bubble stability have been explained ${ }^{12,13,17}$ as a consequence of thresholds for bubble instabilities toward shape oscillations and diffusive processes, in very good agreement with experiment; cf. e.g., the results in Refs. 2, 22-24, and 34. Likewise, the long-standing puzzle of why a small percentage of noble gas in the gas mixture used in experiment is necessary for stable SBSL ${ }^{35}$ was solved ${ }^{14}$ by accounting for chemical reactions in the bubble, which deplete the bubble interior of all gases except noble gases. This argon rectification theory ${ }^{14}$ has meanwhile been confirmed in various experiments. ${ }^{23,24,36}$ All these conclusions were drawn on the basis of the very simple bubble dynamical description sketched above, with a constant $\gamma=1$, as the size of the bubble is so small that heat is readily exchanged between its interior and exterior for most of the cycle, resulting in isothermal behavior.

The importance of the different material parameters has been investigated numerically ${ }^{13,16,18,37}$ and analytically. ${ }^{17}$ The dependence of viscosity, gas solubility, and water vapor pressure on water temperature conspire to allow for larger maximal SBSL light intensities at lower water temperatures. ${ }^{16,38}$ Further studies ${ }^{17,37}$ elucidated the possibilities for achieving SL in larger bubbles or with more violent collapses ("upscaling').

Whereas all these facts fit together to form a consistent 
picture of bubble dynamics, the process of light emission is still not included. The most simple explanation, namely, thermal blackbody radiation, has been discarded on two accounts: (i) blackbody radiation would be coupled to the dynamics of the temperature in the bubble, i.e., it is expected to change on the time scales of bubble dynamics, which from theoretical calculations seem to be longer than the observed pulse widths. ${ }^{39-42}$ (ii) Gompf et al., ${ }^{39,40}$ and later Hiller et al. ${ }^{41}$ experimentally found the pulse width to be independent of the wavelength $\lambda$ of the radiation. Moran and Sweider ${ }^{42}$ find only a rather weak dependence $\lambda$ in some parameter regimes. However, a blackbody emitter would yield much longer pulses in the red than in the ultraviolet, unlike what is seen in these experiments; see Sec. III.

Our aim in this paper is to capture the essentials of the light production mechanism by a simple, but quantitative, model along the lines of the above-mentioned bubble dynamical approach. With the uniform bubble interior used in our calculations, the light emission must necessarily be determined by the uniform temperature $T(t)$ inside the bubble. In Sec. II we will deal with the consistent computation of $T(t)$ from $R(t)$. We proceed by presenting results confirming the failure of ideal blackbody calculations to account for the light emission characteristics (Sec. III). The full model contains a temperature- and wavelength-dependent opacity of the gas (Sec. IV). In Sec. V a systematic comparison of the theoretical results to experimental data is made, which turns out to be rather satisfactory. In Sec. VI we present predictions made and conclusions drawn from our model.

\section{FROM BUBBLE DYNAMICS TO TEMPERATURE ESTIMATES}

\section{A. Bubble dynamics near collapse}

Even for bubble oscillations of a strongly nonlinear character (involving large changes in the bubble radius and violent collapses), the Rayleigh-Plesset equation provides an accurate description as long as the speed of the bubble wall $|\dot{R}|$ is smaller than the speed of sound in water, $c_{l}$ $=1481 \mathrm{~ms}^{-1} \cdot{ }^{43}$ Although this condition breaks down in the last ns before the collapse ends (i.e., before the minimum radius $R_{\min }$ is reached), it is reestablished quickly as the bubble decelerates and starts its reexpansion, which is generically much slower $\left(\sim 100 \mathrm{~ms}^{-1}\right)$. Therefore, although the details of the time interval around $R \approx R_{\min }$ will not be described very accurately, the orders of magnitude of the bubble dynamical quantities (and also the gas temperature following from these) should be reliable. Especially, the asymmetric shape of the collapse displayed in Figs. 1(a) and 1(b) not only occurs in the calculated solution of (1), but is also seen experimentally. ${ }^{40,44,45}$

\section{B. Including temperature in the Rayleigh-Plesset picture}

We are looking for a self-consistent extension of (1) and (2), including the gas temperature $T$ to form a three-variable ODE system for $R, \dot{R}$, and $T$. Using the excluded volume van der Waals equation of state,

$$
p_{\text {gas }} \frac{4 \pi}{3}\left(R^{3}-h^{3}\right)=\frac{4 \pi}{3} R_{0}^{3} v_{m} \mathcal{R} T,
$$

with the ideal gas constant $\mathcal{R}$ and the specific molar volume $v_{m}$ under normal conditions, (2) yields

$$
\dot{T}=-[\gamma(R, \dot{R}, T)-1] \frac{3 R^{2} \dot{R}}{R^{3}-h^{3}} T .
$$

To close the system of equations, we have to specify $\gamma$. Effectively, $\gamma$ is meant to describe the interplay of temperature changes because of bubble dynamics (expansion or compression of the gas) on the one hand and temperature changes because of thermal conduction on the other hand. If e.g. the bubble motion (time scale $\tau_{\text {dyn }}$ ) is faster during collapse than the time scale of heat conduction through the bubble $\tau_{\text {th }}$, the collapse will be (nearly) adiabatic and $\gamma \approx \Gamma$, where $\Gamma=5 / 3$ is the adiabatic exponent for argon. Away from collapse, however, the bubble wall motion is relatively slow and heat conduction acts faster than the bubble motion, so that the bubble is (nearly) isothermal, with $\gamma \approx 1$. An order of magnitude estimate for $\tau_{\text {th }}$ is $R^{2} / \chi_{0}$, where $\chi_{0}$ is the thermal diffusivity of the gas inside the bubble under ambient conditions. With $\chi_{0} \approx 2.2 \times 10^{-5} \mathrm{~m}^{2} / \mathrm{s}$ for argon and $R$ of the order of a few $\mu \mathrm{m}$, we find that $\tau_{\mathrm{th}} \sim 100 \mathrm{~ns}$, which is both much longer than the crucial time scale for light emission (so that in this regime the bubble can be treated adiabatically) and much shorter than the driving time scale (so that isothermality holds through most of the oscillation cycle).

The presence of heat conduction modifies the results of the RP calculations in two ways: not only is $\gamma$ varying in time, but the heat conduction leads to the formation of a thermal boundary layer at the bubble wall, effectively diminishing the radius of high-temperature gas. Both effects will be discussed in the following.

\section{Computation of $\gamma$}

For the limit of weak (linear) bubble oscillations, the time dependence of the effective polytropic exponent $\gamma$ is known: Prosperetti ${ }^{32}$ has derived a rigorous, analytical formula that relates $\gamma$ to the Péclet number Pe. This number, by definition, measures the relative importance of heat advection and diffusion. Instead of using the rather complicated equation in Ref. 32 in its full form, we use a fit that reproduces its shape very closely, namely,

$$
\gamma(\mathrm{Pe})=1+(\Gamma-1) \exp \left(-\frac{A}{(\mathrm{Pe})^{B}}\right),
$$

with numerical parameters $A \approx 5.8, B \approx 0.6$. Obviously, $\gamma(\mathrm{Pe} \rightarrow 0) \rightarrow 1$ (isothermal behavior, where thermal diffusion is dominant) and $\gamma(\mathrm{Pe} \rightarrow \infty) \rightarrow \Gamma=\frac{5}{3}$ (adiabatic behavior, where advection is dominant), as it is required. While Prosperetti's formula was developed for weak bubble oscillations, it does retain its physical meaning of interpolating between isothermal and adiabatic behavior in the case of nonlinear oscillations. Therefore, we venture to extend its application here to the strong collapses of SL bubbles, using a time-dependent, instantaneous Péclet number, 


$$
\mathrm{Pe}=\operatorname{Pe}(t)=R(t)|\dot{R}(t)| / \chi(R, T),
$$

which can be obtained from a dimensional argument or simple estimates of the terms in the PDE of temperature conduction. Pe changes over several orders of magnitude during a typical RP oscillation. For a very large part of the driving cycle, the bubble follows the relatively slow time scales of the driving, yielding small Péclet numbers. Significant deviations from isothermal behavior only occur in the vicinity of the collapse, where (7) will yield large Pe and, consequently, a strong temperature increase under compression.

\section{Dependence of $\chi$ on state variables}

As the argon bubble becomes very dense at collapse (reaching densities of solid state matter), we use the Enskog theory of dense gases (cf. e.g., Ref. 46) to compute the dependence of $\chi$ on $R$ and $T$. The result is

$$
\chi(R, T)=\frac{25}{48} \Gamma^{-1}\left(\frac{\pi a_{\mathrm{Ar}}^{2} \mathcal{R} T}{\mu_{\mathrm{Ar}}}\right)^{1 / 2} G(x),
$$

with the molecular weight of argon $\mu_{\mathrm{Ar}} \approx 0.040 \mathrm{~kg} / \mathrm{mol}$, and the effective atomic diameter $a_{\mathrm{Ar}} \approx 0.34 \mathrm{~nm} .{ }^{46}$ Here $G(x)$ is a function of the dimensionless density,

$$
x=\frac{2 \pi N_{\mathrm{A}} a_{\mathrm{Ar}}^{3}}{3 v_{m}} \frac{R_{0}^{3}}{R^{3}},
$$

where, $N_{\mathrm{A}}$ is Avogadro's number. The virial expansion approximation used in Enskog theory yields

$$
\begin{aligned}
G(x)= & \frac{1}{x}\left(\frac{1}{1+c_{1} x+c_{2} x^{2}+c_{3} x^{3}}+1.2 x\right. \\
& \left.+0.755 x^{2}\left(1+c_{1} x+c_{2} x^{2}+c_{3} x^{3}\right)\right),
\end{aligned}
$$

with the virial coefficients $c_{1}=0.625, c_{2}=0.2869, c_{3}$ $=0.115$. Note that, in the limit of small densities, these formulas recover the well-known proportionalities $\chi^{\propto} \sqrt{T} / \rho_{g}$, where $\rho_{g}$ is the gas density.

The system of equations (1), (4)-(10) constitutes a closed ODE system for $R, \dot{R}$, and $T$. In the next two paragraphs we will discuss slight modifications of this model.

\section{E. Adiabatic and isothermal conditions around collapse}

The condition of adiabaticity around collapse is violated according to the equations presented above when the bubble decelerates and the velocity changes sign, giving $\mathrm{Pe}=0$ and therefore $\gamma=1$ for $\dot{R}=0$. Thus, $\gamma(t)$ looks as displayed in Fig. 1(c) (dashed line): after rising toward $\Gamma$ during collapse, it drops to 1 and then rises again until it finally drops back to the isothermal value as the bubble expands. This is an unphysical feature of the present model, because the computation of Pe from $R(t), \dot{R}(t)$ does not account for the fact that the hot bubble cannot cool instantaneously. To get rid of this behavior, $\gamma$ is kept at its maximum value during the period of fall and rise around the instant where $\dot{R}=0$ [Fig. 1(c) solid

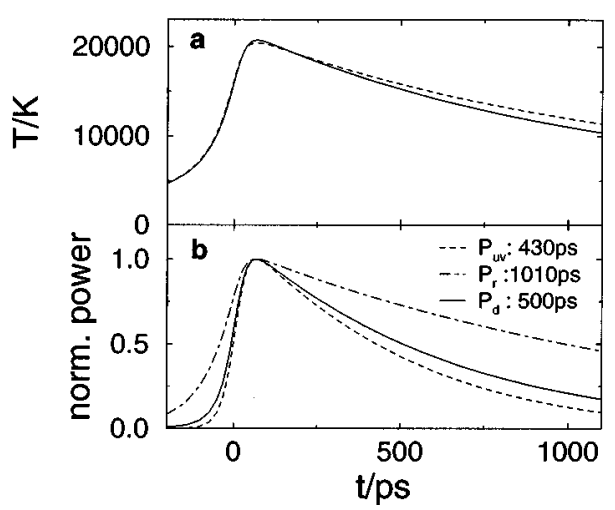

FIG. 2. (a) Temperature pulse $T(t)$ according to (11), as resulting from the dynamics displayed in Fig. 1 , i.e., $f=20 \mathrm{kHz}, P_{a}=1.3 \mathrm{~atm}$, and $R_{0}$ $=5.0 \mu \mathrm{m}$. The uniform gas in the bubble reaches a maximum temperature of $T_{\max } \approx 20000 \mathrm{~K}$. The dashed line gives the virtually unaltered curve without the modification in $\gamma$. (b) Light intensities emitted by an ideal blackbody argon bubble with $T(t)$ as in (a), for three different wavelength intervals. Shown are the total power $P_{\mathrm{UV}}$ in the UV range $(200 \mathrm{~nm}<\lambda<300 \mathrm{~nm}$, dashed), the red $P_{r}(700 \mathrm{~nm}<\lambda<800 \mathrm{~nm}$, dot-dashed), and the complete detectable spectrum $P_{d}(200 \mathrm{~nm}<\lambda<800 \mathrm{~nm}$, solid $)$. All pulses have been normalized to their respective maximum heights. The computed peak powers in absolute units are $1.7 \times 10^{-2} \mathrm{~W}$ for $P_{\mathrm{UV}}, 6.7 \times 10^{-4} \mathrm{~W}$ for $P_{r}$, and $3.2 \times 10^{-2} \mathrm{~W}$ for $P_{d}$. The total number of emitted photons is $4.4 \times 10^{7}$; the FWHM of the pulses is indicated in (b).

line]. This is just a cosmetic correction, as numerical tests have shown that the computed light intensity is modified only on a $10 \%$ level by this modification, the bubble dynamics and temperatures being almost identical [cf. Figs. 1 and 2(a)].

\section{F. Thermal cooling in the boundary layer and the bulk gas}

In the uniform bubble model, the gas in the collapsing bubble becomes much hotter than the water outside, so that there should be a vigorous exchange of heat, which generates a cool thermal boundary layer inside the bubble wall. The equations presented so far only capture the bulk effects of cooling by expansion.

If a hot sphere is instantaneously brought into contact with an infinite liquid, the solution of the heat conduction equation can be found analytically (cf. Ref. 47), suggesting a thermal boundary layer of thickness $\delta_{\mathrm{th}}(t)=2[\chi(t)(t$ $\left.\left.-t_{0}\right)\right]^{1 / 2}$ for $t>t_{0}$. Here, $\delta_{\text {th }}$ grows, starting from 0 at a certain time $t_{0}$, reflecting the time it takes the bubble to establish a sufficiently high Péclet number after the onset of collapse. While $t_{0}$ is in this way somewhat ambiguously defined, we notice that on the time scales of our interest for light emission $(\sim 100 \mathrm{ps})$, we have typically $\delta_{\mathrm{th}} \sim 10 \mathrm{~nm}$, so that this boundary layer process does not modify the energy of the bubble interior markedly (note that even for small bubbles with $R_{0}=2 \mu \mathrm{m}$, the van der Waals hard core radius is still $>200 \mathrm{~nm}$ ). Indeed, a numerical check shows that taking $\delta_{\text {th }}$ into account leads to only very minor modifications of the temperature versus time curve. Therefore, we will not include it into the computations here. 
Thermal cooling of the whole bubble will, however, be accounted for by adding a diffusive heat loss term to (5), i.e., the full formula we use for the temperature change is

$$
\dot{T}=-[\gamma(R, \dot{R}, T)-1] \frac{3 R^{2} \dot{R}}{R^{3}-h^{3}} T-\chi \frac{T-T_{\infty}}{R^{2}} .
$$

The time scale $R^{2} / \chi$ is greater or equal to $\sim 10 \mathrm{~ns}$, so that this cooling has no effect on the fast dynamics during collapse or on the temperatures and light intensities computed. It only reduces the temperature back to the ambient (water) temperature $T_{\infty}$ during the afterbounce phase of the oscillation and ensures that the average energy content of the bubble stays constant from cycle to cycle.

\section{G. Resulting temperature pulses}

The system of equations (1), (4), (6)-(10), (11) is the final version of our model. We use a standard Runge-Kutta adaptive step size algorithm to solve it numerically for $R(t)$, $\dot{R}(t)$, and $T(t)$. Figure 2(a) shows the temperature pulse computed using the present model for the example parameters from Fig. 1, yielding a maximum temperature of $T_{\max }$ $\approx 20000 \mathrm{~K}$, which is in accord with many previous theoretical predictions and experimental conclusions, e.g., Refs. 2, 4. It is obvious that it reflects the asymmetry of the bubble collapse dynamics: the temperature rise time is much smaller than its fall time; the total FWHM is $\approx 1100$ ps here. This is comparable to the turnaround time of $R(t)$. Omitting the modification in $\gamma$ discussed in Sec. II E leaves $T(t)$ virtually unchanged [the dashed line in Fig. 2(a)].

\section{THE FAILURE OF THE IDEAL BLACKBODY MODEL}

In the following paragraphs, we will treat the ideal blackbody light emission from bubbles with temperatures computed from the above model. Although we show that the assumption of an ideal blackbody is insufficient to explain the experimental data, we present these results because many elements of the refined model presented later in Sec. IV have to be introduced already here.

\section{A. Local thermodynamic equilibrium and blackbody radiation}

First, we have to make sure that the time scales of bubble dynamics we have encountered here (at least a few $10^{-11} \mathrm{~s}$ ) are sufficient to allow for local thermodynamic equilibration of the bulk gas. The collision time scale in gases of the densities and temperatures we are dealing with here is comparable to that in liquids, and will therefore be as short as $\sim 10^{-13} \mathrm{~s},{ }^{46}$ corresponding to typical intermolecular distances of $10^{-10} \mathrm{~m}$ and typical particle velocities of $10^{3} \mathrm{~m} / \mathrm{s}$. That is, there is enough time for the atoms to undergo several collisions in order to attain local thermodynamic equilibrium (LTE). One can therefore assume that every volume element of gas in the bubble is in thermal equilibrium with its surroundings.

Necessarily, an ensemble of atoms at a well-defined temperature will have a radiation source function (the intensity of emitted radiation without taking into account the interac- tion of the emitted photons and the atoms) corresponding to a Planck blackbody radiation spectrum. The source spectral intensity (energy per unit time, wavelength interval, solid angle, and projected surface area) at wavelength $\lambda$ is thus given by Planck's law,

$$
I_{\lambda}^{\mathrm{Pl}}[T]=\frac{2 h c^{2}}{\lambda^{5}\left[\exp \left(h c / \lambda k_{\mathrm{B}} T\right)-1\right]},
$$

with the Planck and Boltzmann constants $h$ and $k_{\mathrm{B}}$, and the speed of light in vacuum $c$. The emission changes with time according to the temperature changes $T(t)$. In order to calculate the actually observed radiation, however, we have to take into account the degree of absorption of the emitted photons in the radiating body (the hot gas bubble) itself, i.e., its opacity or optical thickness. In this section we consider the idealized blackbody approximation, where the bubble is considered as optically thick and absorbs perfectly (its opacity is infinite). Accordingly, it emits light from a thin surface layer only, since the radiation originating in the bulk of the bubble is reabsorbed before it can reach the surface.

\section{B. Calculating the properties of the pulses}

For the optically thick case, the emission is completely described by the blackbody radiation equation (12). Together with $T(t)$ calculated according to Sec. II, this yields a time series for the emitted intensity at wavelength $\lambda, I_{\lambda}(t)$ $=I_{\lambda}^{\mathrm{Pl}}[T(t)]$, as well as for the spectral radiance (emitted energy per time and wavelength interval) obtained by integrating over the projected bubble surface and all solid angles:

$$
P_{\lambda}^{\mathrm{Pl}}(t)=4 \pi^{2} R(t)^{2} I_{\lambda}^{\mathrm{Pl}}[T(t)] .
$$

In the case of SBSL, only a certain part of the spectrum is detectable. The spectral radiance decreases strongly toward the red, and the major part of the energy is captured in the wavelength range $\lambda<\lambda_{r} \approx 800 \mathrm{~nm}$. On the other hand, the water surrounding the bubble strongly absorbs UV light below $\lambda_{\mathrm{UV}} \approx 200 \mathrm{~nm}$, so that this portion of the emitted light is lost, and what is measured as the total power of the pulse outside the flask is the integral,

$$
P^{\mathrm{Pl}}(t)=\int_{\lambda_{\mathrm{UV}}}^{\lambda_{r}} P_{\lambda}^{\mathrm{Pl}}(t) d \lambda .
$$

It is of special interest to look at the temporal length of the light pulses in different wavelength intervals. In several experiments these pulse widths have been measured with filters of bandwidth $\Delta \lambda \approx 100 \mathrm{~nm}^{39}$ and $\Delta \lambda \approx 40 \mathrm{~nm},{ }^{42}$ or alternatively with a spectrographic procedure. ${ }^{41}$ While Gompf et $a l .{ }^{39}$ and Hiller et $a l .{ }^{41}$ could not detect any spectral variation of the pulse width, a small increase toward the red wavelengths is reported by Moran and Sweider. ${ }^{42}$

Within the present formalism, it is easy to integrate the spectral radiance over suitable wavelength intervals and to compare with the experimental results.

\section{Blackbody approximation of bubble radiation}

Figure 2(b) shows a simulation of the light pulses from a strongly collapsing bubble treated as a blackbody (param- 
eters $P_{a}, R_{0}$ as in Fig. 1). The pulse of total power emitted into the visible part of the spectrum $\left[P_{d}\right.$ in Fig. 2(b)] reflects the characteristic asymmetry of the dynamics, i.e., the fall time of the emitted radiance is longer than the rise time. ${ }^{40}$ The "black" bubble's pulse width, however, is well outside the range of experimental observations ${ }^{39-42}$-with $\approx 500 \mathrm{ps}$ it is too long. While shorter pulses can be obtained with smaller driving pressures and radii, e.g., $\approx 275$ ps FWHM with $P_{a}=1.2 \mathrm{~atm}$ and $R_{0}=3.0 \mu \mathrm{m}$, the average pulse widths are clearly overestimated in the blackbody model.

There are two more serious shortcomings of this calculation, namely, (i) it fails to reproduce quantitatively the absolute magnitude of the pulses, and (ii) it predicts a too strong variation of the pulse width with the photon wavelength interval. For the example of Fig. 2, the total number of photons emitted over one period is $\approx 4.4 \times 10^{7}$ photons, about ten times as many as observed in the strongest light pulses in argon experiments. ${ }^{2,38,48}$ The variation of the pulse width with $\lambda$ is shown in Fig. 2(b), where the pulse intensities in the ultraviolet wavelength interval $\left(P_{\mathrm{UV}}, 200-300\right.$ $\mathrm{nm})$, in the red portion of the spectrum $\left(P_{r}, 700-800 \mathrm{~nm}\right)$, and in the whole detectable spectral range $\left(P_{d}, 200-800\right.$ $\mathrm{nm})$ are depicted. The "red" pulse is more than twice as long as the "ultraviolet" pulse, a variation much too large to be compatible with the observations in Refs. 39-42. The variations do not become significantly smaller if smaller $P_{a}$ and/or $R_{0}$ are analyzed. This indicates that the assumption of the bubble as a simple blackbody radiation source needs refinement.

\section{FROM TEMPERATURE TO LIGHT EMISSION: INCORPORATING FINITE OPACITY}

\section{A. The bubble as a volume emitter}

We have assumed in the previous section that the bubble emits as an ideally absorbing blackbody with infinite opacity. Comparing to more elaborate simulations, we see that this may not be the case: in an important study by Moss et al., ${ }^{4}$ where shock waves inside the bubble play a crucial role and heat the gas to central temperatures above $10^{5} \mathrm{~K}$, finite opacity is included in the calculations of their model. Although no accurate experimental data on the absorption coefficient of gases in the relevant temperature and density range is available, the modeling in Ref. 4 suggests that practically the whole bubble is transparent (optically thin) for almost all the time. This means that the bubble has to be treated as a volume emitter. The detected radiation does not come just from a thin surface layer, but from the whole volume, as the photons are only weakly absorbed inside the bubble.

Let us denote the photon absorption coefficient (the inverse of the absorption length), depending on the wavelength and, via temperature, on the location within the bubble and the time, by $\kappa_{\lambda}[T(s, t)]$. Then, the emitted intensity at wavelength $\lambda$, after traveling a distance $s$ in the medium, becomes $^{49,50}$

$$
\begin{aligned}
I_{\lambda}(s, t)= & \int_{0}^{s} \kappa_{\lambda}\left[T\left(s^{\prime}, t\right)\right] \\
& \times \exp \left(-\int_{0}^{s^{\prime}} \kappa_{\lambda}\left[T\left(s^{\prime \prime}, t\right)\right] d s^{\prime \prime}\right) I_{\lambda}^{\mathrm{Pl}}\left[T\left(s^{\prime}, t\right)\right] d s^{\prime} .
\end{aligned}
$$

Note that the source function is still $I_{\lambda}^{\mathrm{Pl}}$, because of LTE. Keeping the approximation of uniform temperature throughout the whole bubble, this reduces to

$$
I_{\lambda}(s, t)=I_{\lambda}^{\mathrm{Pl}}[T(t)]\left(1-\exp \left\{-\kappa_{\lambda}[T(t)] s\right\}\right), \quad 0<s<2 R .
$$

In the limit of an optically thick blackbody $\left(\kappa_{\lambda} \rightarrow \infty\right)$, this is $I_{\lambda}^{\mathrm{Pl}}(t)$, while for small $\kappa_{\lambda}$ (the transparent limit) the emitted intensity is considerably reduced, becoming proportional to the dimensionless absorptivity $\kappa_{\lambda} s$. A very weakly absorbing body is a very weak emitter. To get the total emitted power, the intensity (16) (per surface area and per solid angle) must first be integrated over the cross section of the emitting part of the bubble, the thickness $s$ of the medium varying as $s=2 R \cos \theta$ with the angle $\theta$ from the line of view. This gives the emitted power from the total bubble per unit solid angle. As we assume isotropy, this quantity multiplied by $4 \pi$ is the total emitted power per wavelength interval:

$$
\begin{aligned}
P_{\lambda}(t) d \lambda= & 4 \pi^{2} R^{2} I_{\lambda}^{\mathrm{Pl}}[T(t)]\left(1+\frac{\exp \left(-2 \kappa_{\lambda} R\right)}{\kappa_{\lambda} R}\right. \\
& \left.+\frac{\exp \left(-2 \kappa_{\lambda} R\right)-1}{2 \kappa_{\lambda}^{2} R^{2}}\right) d \lambda .
\end{aligned}
$$

In the transparent limit $\left(2 \kappa_{\lambda} R \ll 1\right)$, this yields

$$
\begin{aligned}
P_{\lambda}^{\text {trans }}(t) d \lambda & =4 \pi \kappa_{\lambda} I_{\lambda}^{\mathrm{Pl}}[T(t)] \frac{4 \pi R^{3}}{3} d \lambda \\
& =\frac{4}{3} \kappa_{\lambda} R P_{\lambda}^{\mathrm{Pl}}(t) d \lambda,
\end{aligned}
$$

which nicely illustrates the features of a volume emitter, multiplying $I_{\lambda}^{\mathrm{Pl}}$ by the volume of the bubble and reducing the emission as compared to the blackbody limit through multiplication with $\kappa_{\lambda}$. Obviously, for $2 \kappa_{\lambda} R \gg 1$, Eq. (17) yields the emitted power $P_{\lambda}^{\mathrm{Pl}}(t) d \lambda$ from an ideal blackbody, proportional to the surface area [cf. also (13)].

\section{B. Photon absorption in a weakly ionized gas}

To determine the absorption coefficient, and thus via (17) the observable emission, we have to find out the dominant (microscopic) photon absorption processes in the gas. This is not an easy task, as pointed out in Ref. 4, mostly because the combination of high temperatures and large densities inside the collapsed bubble are not encountered in other physics experiments, so that almost no data are available. Nevertheless, some general formulas are applicable. Evaluating the Saha equation for temperatures of several $10^{4} \mathrm{~K}$ shows that the gas (argon) is only weakly ionized (i.e., the number density of electrons $n_{e}$ is much smaller than that 
of neutral atoms $n$ ), because $k_{\mathrm{B}} T \ll E_{\text {ion }}$, the (first) ionization energy of argon being $E_{\mathrm{ion}} \approx 15.8 \mathrm{eV}$. More precisely, the degree of ionization $\alpha=n_{e} / n$ is ${ }^{49}$

$$
\alpha[T]=\left(\frac{2 \pi m_{e} k_{\mathrm{B}} T}{h^{2}}\right)^{3 / 4}\left(\frac{2 u_{+}}{n u_{0}}\right)^{1 / 2} \exp \left(-\frac{E_{\text {ion }}}{2 k_{\mathrm{B}} T}\right),
$$

where $m_{e}$ is the electron mass and $u_{+}, u_{0}$ are the statistical weights for the ionic and the neutral ground state, respectively. Consistent with the hydrogen-like atom model of Ref. 49 , which we will use below to evaluate atomic energy levels, we specify $2 u_{+} / u_{0}=1$ for the statistical weights.

As the maximum temperatures encountered in the SL parameter range are only of the order of a few eV (less than $2 \mathrm{eV}$ in the case of Fig. 2), the degree of ionization, even during the most violent collapses, is still $\alpha \lesssim 1 \%$ for argon. Therefore, the free-free interactions of electrons and ions (inelastic collisions of charged particles) are not necessarily the dominant processes of photon absorption (as it would be the case in every considerably ionized gas ${ }^{49}$ ). We have to compare their contribution to absorption, denoted by $\kappa_{\lambda}^{f f+}$, with the absorption coefficient $\kappa_{\lambda}^{f f 0}$ due to the interaction of photons with electrons colliding inelastically with neutral atoms, for which $\kappa_{\lambda}^{f f 0} \propto \alpha n^{2}$ instead of $\kappa_{\lambda}^{f f+} \propto \alpha^{2} n^{2}$.

According to Ref. 49, we have

$\kappa_{\lambda}^{f f+}[T]=\frac{4}{3}\left(\frac{2 \pi}{3 m_{e} k_{\mathrm{B}} T}\right)^{1 / 2} \frac{Z^{2} e^{6} \lambda^{3}}{\left(4 \pi \epsilon_{0}\right)^{3} h c^{4} m_{e}} \alpha[T]^{2} n^{2}$,

with the effective charge $Z$ of the ions (taken to be $Z=1$ here), the electron charge $e$, and the vacuum permeability $\epsilon_{0}$.

To evaluate $\kappa_{\lambda}^{f f 0}$ we need the effective inelastic collision cross section of electrons with neutral atoms, the so-called transport scattering cross section $\sigma_{\mathrm{tr}} \cdot{ }^{51}$ It is normally of the order of (mostly somewhat larger than) the geometrical cross section of the neutral atoms. The quantity $\sigma_{\text {tr }}$ is tabulated as a function of the kinetic energy $E_{e}$ of the incident electron; with good accuracy, we have for argon a linear dependence of the cross section on $E_{e},{ }^{52}$

$$
\sigma_{\mathrm{tr}}\left(E_{e}\right) \approx c_{\mathrm{tr}} E_{e}+d_{\mathrm{tr}},
$$

in the relevant range of electron energies, with the constants $c_{\mathrm{tr}} \approx 1.6 \times 10^{-20} \mathrm{~m}^{2} / \mathrm{eV}, d_{\mathrm{tr}} \approx-0.6 \times 10^{-20} \mathrm{~m}^{2}$.

Using the linearity of this formula, we arrive at an expression for $\kappa_{\lambda}^{f f 0}\left[v_{e}\right]$ for interacting electrons of velocity $v_{e}=\sqrt{2 E_{e} / m_{e}}$, namely,

$$
\kappa_{\lambda}^{f f 0}\left[v_{e}\right]=\frac{e^{2} \lambda^{2}}{\left(4 \pi \epsilon_{0}\right) \pi c^{3}} \alpha[T] n^{2}\left(c_{\mathrm{tr}} v_{e}^{3}+\frac{4 d_{\mathrm{tr}} v_{e}}{3 m_{e}}\right) .
$$

Equation (22) uses the long wavelength approximation $h c / \lambda k_{\mathrm{B}} T<1$, but should yield reasonable values for smaller wavelengths, too. ${ }^{49}$ Inserting (19) in (22) and averaging the equation over the (Maxwell-distributed) electron velocities, we obtain the absorption coefficient as a function of temperature,

$$
\begin{aligned}
\kappa_{\lambda}^{f f 0}[T]= & \frac{e^{2}}{\pi \epsilon_{0}} \frac{\left(2 k_{\mathrm{B}} T\right)^{9 / 4} n^{3 / 2}}{h^{3 / 2} c^{3} m_{e}^{3 / 4} \pi^{3 / 4}} \lambda^{2}\left(c_{\mathrm{tr}}+\frac{d_{\mathrm{tr}}}{3 k_{\mathrm{B}} T}\right) \\
& \times \exp \left(-\frac{E_{\text {ion }}}{2 k_{\mathrm{B}} T}\right) .
\end{aligned}
$$

Numerical computation of (23) and a comparison to the ionic contribution shows that (for argon) $\kappa_{\lambda}^{f f 0}$ is indeed comparable to $\kappa_{\lambda}^{f f+}$ in most regions of the SL parameter range.

Photonic bound-free ionization of already excited atoms and bound-bound excitations of electrons on the discrete energy levels of the atom in question also contribute to the photon absorption. In both cases the discrete energy levels of the neutral atom must be known or modeled. For the boundfree processes, we follow the "hydrogen-like atom" model of Ref. 49, which results in

$$
\begin{aligned}
\kappa_{\lambda}^{b f}[T]= & \frac{64 \pi^{4}}{3 \sqrt{3}} \frac{Z^{4} e^{10} m_{e} n}{\left(4 \pi \epsilon_{0}\right)^{5} h^{6} c^{4}} \lambda^{3} \\
& \times \sum_{j=j^{*}}^{\infty} \frac{1}{j^{3}} \exp \left(-\frac{E_{\text {ion }}-E_{j}}{k_{\mathrm{B}} T}\right),
\end{aligned}
$$

where $j$ is the index of the bound states $(j=1$ being the ground state), and $E_{j}=E_{\text {ion }} / j^{2}$ the corresponding energies, with $E_{j}$ increasing from $E_{\infty}=0$ to $E_{1}=E_{\text {ion }}$. The sum starts at the lowest-lying level $j^{*}$ for which $E_{j^{*}}<h c / \lambda$. Adding this absorption to $\kappa_{\lambda}^{f f+}$ (which is also due to the presence of ions), we get the total ionic absorption coefficient $\kappa_{\lambda}^{\text {ion }}$.

The atomic level system for argon (and the other noble gases as well) features a large gap between the ground state and the next-lowest level (described here by $j=2$ ). Above $j=2$, the term system has a lot of relatively closely spaced energy levels, which suggests a continuum model for $\lambda$ $>\lambda_{2}$, where $E_{2}=h c / \lambda_{2}$. Indeed, with these assumptions the sum over $j$ in (24) can be replaced by an integral and computed analytically. ${ }^{49}$ Inserting $\alpha$ from (19), we finally get the relatively simple formula,

$$
\begin{aligned}
\kappa_{\lambda}^{\mathrm{ion}}[T]= & \frac{16 \pi^{2}}{3 \sqrt{3}} \frac{e^{6} k_{\mathrm{B}} T n}{\left(4 \pi \epsilon_{0}\right)^{3} h^{4} c^{4}} \lambda^{3} \\
& \times \exp \left(-\frac{E_{\text {ion }}-h c / \max \left\{\lambda, \lambda_{2}\right\}}{k_{\mathrm{B}} T}\right) .
\end{aligned}
$$

The wavelength $\lambda_{2}$ corresponding to the lowest-lying level above the ground state can be retrieved from tables, e.g., in Ref. 53.

The bound-bound processes are harder to model. The absence of emission or absorption lines in the SL spectra ${ }^{2,54}$ suggests that the line contributions are either very weak and swamped by the continuum spectrum, or suffer so much line broadening that they form part of the continuum. ${ }^{55}$ While we cannot decide this case at present, we remark that inserting the maximum values of temperature and pressure from the presented calculations into the well-known formulas for pressure broadening (the most plausible candidate for extensive line broadening in our situation; cf., e.g., Ref. 50, 56) gives considerable, but not excessively large, line widths, so that a line structure would still be visible in the spectrum. Since they are not observed, we neglect the bound-bound contributions here, bearing in mind that, by only considering the absorption from (23) and (25), we have underestimated the optical thickness and therefore the emitted power from the bubble. Moreover, we do not account for impurities in the argon bubble due to the presence of a small percentage of 
molecular gases and/or water vapor. The presence of these more easily excitable species could cause a larger opacity, too. ${ }^{57,58}$

Although the formulas for the contributions to absorption may look lengthy, they are easily implemented into the formalism and present no computational difficulties. We can now straightforwardly compute the temperature and wavelength dependence of $\kappa_{\lambda}[T]=\kappa_{\lambda}^{f f 0}[T]+\kappa_{\lambda}^{\text {ion }}[T]$. We see from (23) and (25) that it decreases strongly with decreasing temperature, because of the exponential factors in these equations. Therefore, we can expect a fast cutoff in the light intensity when the temperature drops during the reexpansion phase of the oscillation. This will reduce the unrealistically high photon numbers from the ideal blackbody calculations (cf. Sec. III C).

In the next sections, we will use the absorption coefficient $\kappa_{\lambda}[T]$ to compute length and spectral properties of SBSL light pulses from (17). That it is sufficient to compute absorption in order to calculate emission is a consequence of Kirchhoff's law, which states that absorption and emission are reciprocal mechanisms. Thus, the primary processes for the emission of sonoluminescence photons are precisely inverse to those responsible for absorption: (i) bremsstrahlung from inelastic collisions of free electrons with neutral atoms, corresponding to $\kappa_{\lambda}^{f f 0}[T]$; (ii) bremsstrahlung of free electrons in the field of ions $\left(\kappa_{\lambda}^{f f+}[T]\right)$; and (iii) photon emission from the recombination of free electrons and ions to form excited atoms $\left(\kappa_{\lambda}^{b f}[T]\right)$.

\section{Light pulses and spectra in the refined model}

Figure 3 shows the light pulses computed from this model including the calculated opacity due to thermal bremsstrahlung and recombination radiation (again for $P_{a}$ $\left.=1.3 \mathrm{~atm}, R_{0}=5.0 \mu \mathrm{m}\right)$. Let us first look at the dimensionless optical thickness $\tau_{\lambda}(t) \equiv 2 \kappa_{\lambda}[T(t)] R(t)$ of the bubble. In Fig. 3(c) we see that the bubble in this example stays optically thin $\left(\tau_{\lambda}<1\right)$ for the whole oscillation cycle. Figures 3(a) and 3(b) show how different the pulse widths of the temperature $T(t)$ and the total emitted detectable power $P_{d}(t)$ are: the small time interval in which $\tau_{\lambda}$ is relatively large "cuts out" a short window from the temperature pulse to yield intense radiation for only about 150 ps. The light emission quenches very quickly as soon as the temperature drops by just $10 \%$ or so of its maximum value. The physical origin of this is the exponential dependence of the absorption coefficients on the ratio $E_{\text {ion }} / k_{\mathrm{B}} T$, which, moreover, takes on large values because of the huge $E_{\text {ion }}$ for noble gases, so that the variation of opacity with $T$ is enormous.

Comparing the pulse width in the UV and red wavelength regions [Fig. 3(b)], we see that the pulse width differences that were present in the ideal blackbody model have almost disappeared, the "red" pulse centered around $\lambda$ $=750 \mathrm{~nm}$ being only $\approx 10 \%$ longer than the UV pulse. A small difference like this, amounting to $\approx 16$ ps here (at very different absolute intensities), can hardly be detected in today's experiments. ${ }^{40-42}$ Moran and Sweider ${ }^{42}$ do find longer pulses in the red, but only for lower water temperatures, where the bubbles can be driven at larger $P_{a}$ and $R_{0}$ (cf. Ref.

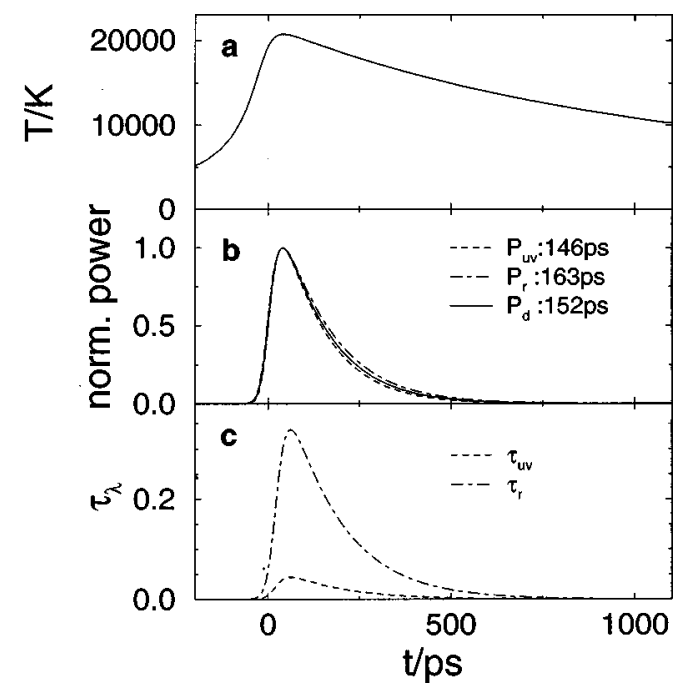

FIG. 3. (a) Temperature pulse $T(t)$ in an argon bubble, identical with the solid line of Fig. 2(a). (b) Resulting light intensities emitted by an argon bubble with a wavelength- and temperature-dependent photon absorption coefficient for different wavelength intervals (parameters as in Fig. 2). Again, $P_{\mathrm{UV}}, P_{r}$, and $P_{d}$ are shown. Here, the variation of the indicated pulse widths is small (only $\approx 10 \%$ with respect to the red pulse). The peak powers are $4.62 \times 10^{-4} \mathrm{~W}\left(P_{\mathrm{UV}}\right), \quad 1.34 \times 10^{-4} \mathrm{~W}\left(P_{r}\right)$, and 1.69 $\times 10^{-3} \mathrm{~W}\left(P_{d}\right)$; this results in a total of $\approx 6.9 \times 10^{5}$ detectable emitted photons. (c) Optical thickness $\tau \equiv\left(2 \kappa_{\lambda} R\right)(t)$ at the center of the UV $(250 \mathrm{~nm}$, dashed) and red (750 nm, dot-dashed) spectral intervals. The bubble is clearly an optically thin (transparent) volume emitter throughout the oscillation (even at collapse), but is absorbing red light considerably stronger than UV photons.

16). This is completely in accordance with the present model, as will be discussed below.

The wavelength independence of the pulse widths arises from the fact that the temperature dependences of (23) and (25) are dominated by terms varying exponentially with the inverse of the temperature, but practically independent of the wavelength, so that the cutoff occurs for the UV and the red light alike. The spectral intensity of the emitted power for the present choice of parameters is given in Fig. 4(a); displayed is the spectral radiance averaged over one driving period, which is the quantity detected in experiment. In absolute intensity as well as in spectral distribution it compares well to the experimental values. ${ }^{2,35}$ The explicit modeling of the absorption edge for $j=2$ [cf. Sec. IV B, Eqs. (24), (25)] has, however, led to a spectral maximum at $\lambda_{2}(\mathrm{Ar}) \approx 294 \mathrm{~nm}$, which is not clearly resolved in experiment (an observed maximum for argon is generally attributed to the onset of absorption by the water ${ }^{2,54}$ ). A possible explanation is that the level system of argon is modified near collapse because of the close packing of atoms under the high pressure. The actual $\lambda_{2}$ may then be shifted to smaller wavelengths.

How does the spectrum differ for other noble gases? The essential parameter distinguishing them is the ionization energy $E_{\text {ion }}$. For the same $P_{a}$ and $R_{0}$, Fig. 4(a) also shows the spectrum for a xenon bubble, computed with the appropriate changes in material parameters. As in experiment, the emission is much more intense than for argon, and it displays a maximum at $\lambda_{2}(\mathrm{Xe}) \approx 336 \mathrm{~nm}$, which is quite close to the actually measured maximum. ${ }^{2}$ In Fig. 4(b) the variation of 


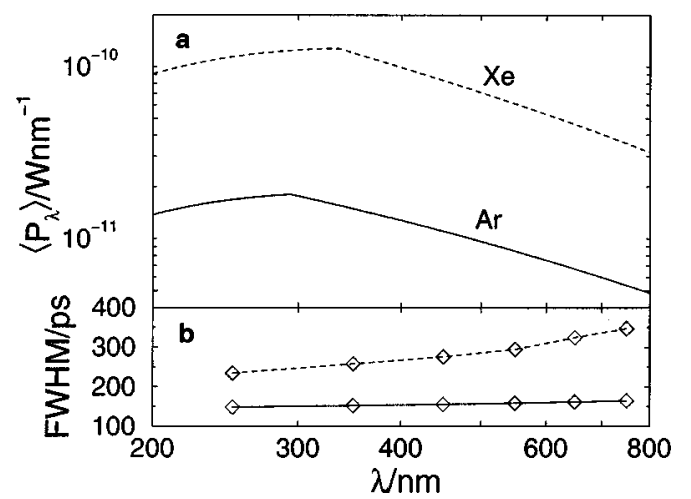

FIG. 4. (a) Spectral radiance of emitted SL light (averaged over one driving period $T_{d}$ ) from an argon bubble with $P_{a}=1.3 \mathrm{~atm}$ and $R_{0}=5.0 \mu \mathrm{m}$ (solid line); the driving frequency is $f=20 \mathrm{kHz}$. A xenon bubble driven at the same parameters yields a much higher spectral radiance (dashed). A comparison with the experimental spectra in Ref. 2 shows good agreement both in intensity and in variation across the detectable part of the spectrum $(200 \mathrm{~nm}<\lambda<800 \mathrm{~nm})$; the experimentally reported spectra decay somewhat faster toward the red. (b) The variation of the pulse width with $\lambda$, computed for the total power contained in intervals of $\Delta \lambda=100 \mathrm{~nm}$, centered around $\lambda=250 \mathrm{~nm}, 350 \mathrm{~nm}, \ldots, 750 \mathrm{~nm}$. This corresponds to experiments using filters of $\approx 100 \mathrm{~nm}$ bandwidth. ${ }^{39}$ The calculation for argon (solid) yields very small variations: those for xenon (dashed) are more pronounced and should be detectable in experiment.

the pulse widths with wavelength is shown for both Ar and Xe. Unlike Ar, Xe displays a moderate variation (though still considerably below what one finds for the ideal blackbody), which should be observable, if bubbles of high intensity (and therefore large pulse widths) can be sufficiently stabilized in the experiment. Both the stronger light emission and the higher variance of the pulse width for Xe are well explained by the smaller ionization energy of xenon, $E_{\text {ion }}(\mathrm{Xe})$ $\approx 12.1 \mathrm{eV}$, which yields smaller exponential damping factors in $\kappa_{\lambda}$ at given $T$, and therefore raises opacity. Thus, xenon bubbles become more similar to the optically thick ideal blackbody discussed in Sec. III than argon bubbles. For argon bubbles, stronger collapses lead to higher temperatures and therefore likewise to a behavior closer to an ideal blackbody due to larger absorptivity via smaller $E_{\text {ion }} / k_{\mathrm{B}} T$. In particular, for larger $P_{a}$ and $R_{0}$ the pulse width variation between the red and ultraviolet pulse becomes more significant. This is corroborated by the experimental data in Ref. 42, where a lower water temperature $T_{\infty}$ (allowing for stronger driving and larger bubbles) leads to measurable variations of the pulse widths.

\section{PARAMETER DEPENDENCE OF LIGHT EMISSION IN THEORY AND EXPERIMENT}

In the last section we have seen that the present interpretation of sonoluminescence is capable of producing pulses of the correct shape, size, intensity, and spectrum. Because of the simplicity of the equations used, we are able to compute these characteristics for the whole parameter space of SL bubbles with very little numerical effort. Therefore, we can show that the numbers reported above for our particular example of $P_{a}$ and $R_{0}$ are not special cases, but fit into a consistent picture.

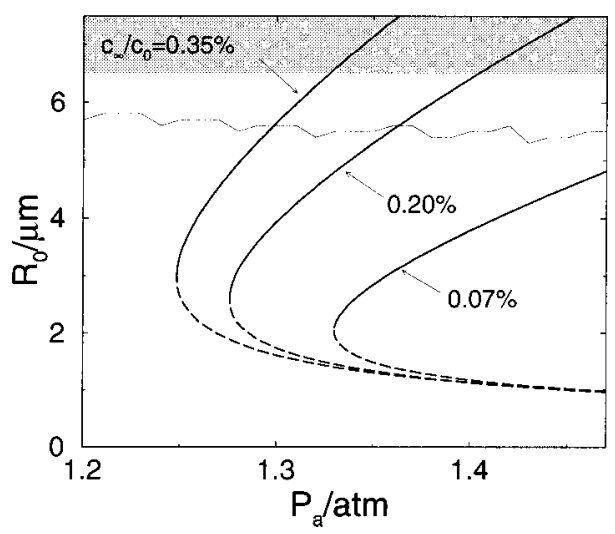

FIG. 5. Curves of diffusive equilibria (thick lines) for argon bubbles driven at $f=20 \mathrm{kHz}$ at dissolved gas concentrations $c_{\infty} / c_{0}=0.07 \%, 0.20 \%$, and $0.35 \%$ (this corresponds to the concentrations reported in Ref. 39). The solid parts of the curves represent stable equilibria $R_{0}^{e}\left(P_{a}\right)$, the dashed parts are unstable. The parametric surface instability restricts the radius to less than $\approx 5.5 \mu \mathrm{m}$ (calculated in Ref. 37, thin solid line) or $\approx 6.5 \mu \mathrm{m}$ (experimental, ${ }^{22}$ indicated as the hatched region).

From studies on diffusive equilibria, ${ }^{13,16,17}$ we know that for stable SBSL there is a unique stable equilibrium ambient radius $R_{0}^{e}$ determined by $P_{a}$ at constant $f$ and $c_{\infty} / c_{0}$ (Fig. 5). Here $R_{0}^{e}\left(P_{a}\right)$ is a monotonically growing function, so that at larger $P_{a}$ the bubbles are not only more strongly driven, but also larger in ambient size. Therefore, the brightest bubbles should be found at the shape instability threshold, which determines the largest bubbles stable toward shape oscillations. The calculations of Ref. 13, which neglect the effect of thermal damping on the bubble oscillation, locate the maximum $R_{0}$ near 5-6 $\mu \mathrm{m}$, almost independent of $P_{a}$, whereas at $P_{a}$ $\gtrsim 1.5 \mathrm{~atm}$, bubbles of all sizes become unstable (RayleighTaylor instabilities) ${ }^{13}$ Experiments ${ }^{22}$ and more elaborate numerical calculations, ${ }^{19}$ which include thermal effects in more detail, have shown that the stability threshold lies somewhat higher, e.g., $7 \mu \mathrm{m} \pm 1 \mu \mathrm{m}$ for $20 \mathrm{kHz}$ driving. ${ }^{22}$ As the ambient water temperature influences viscosity, solubility, and water vapor pressure, the threshold depends also on $T_{\infty}$, allowing for larger $R_{0}$ and larger forcing pressure $P_{a}$ in cooler water. ${ }^{16}$

If the experiment we want to understand is conducted using a gas mixture containing molecular gases, the chemical dissociation theory ${ }^{14}$ predicts that only the monatomic (noble gas) constituents will remain in the bubble. Therefore, the relevant gas concentration $c_{\infty}$ for the experiment is the noble gas partial concentration. If, e.g., air, which contains $1 \%$ argon, is degassed to $20 \%$ of saturation, the actually relevant concentration is $c_{\infty} / c_{0}=0.2 \%$.

Moving along a curve of stable diffusive equilibria at a fixed gas concentration $c_{\infty} / c_{0}$ (Fig. 5), we can compute the fundamental properties of the light emission, namely, pulse widths and light intensities, for all stable SL bubbles at a prescribed gas concentration and driving frequency. The currently available experimental data were obtained at (i) 26.5 $\mathrm{kHz}$ (Ref. 2, only intensities are reported), (ii) $20 \mathrm{kHz}$ (Refs. 39, 40; only pulse widths are reported), (iii) $29 \mathrm{kHz}$ (Ref. 42, 


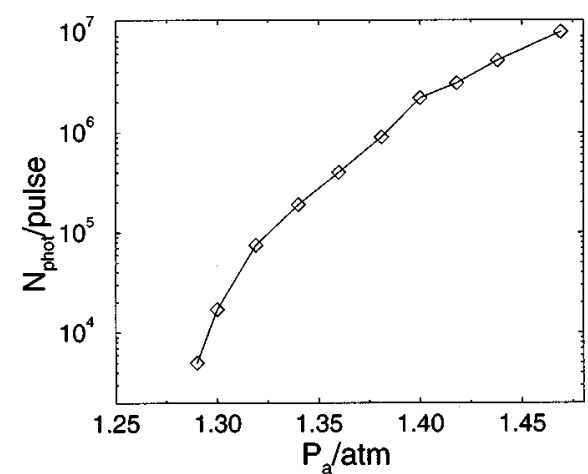

FIG. 6. Calculated total numbers of emitted photons in the detectable wavelength interval $[200 \mathrm{~nm}, 800 \mathrm{~nm}]$ as a function of $P_{a}$, with $R_{0}$ determined by the diffusive equilibrium at $c_{\infty} / c_{0}=0.4 \%$. The driving frequency is 26.5 $\mathrm{kHz}$, as in Ref. 2 (the solid line is a guide to the eye).

where $P_{a}$ is not calibrated), and (iv) $34 \mathrm{kHz}$ (Ref. 41, intensities are given in relative units).

Let us first compare the photon numbers and spectral intensities obtained in (i) to the present model predictions for the given concentration $c_{\infty} / c_{0}=0.4 \%$ : assuming a maximum $R_{0}$ of $7.5 \mu \mathrm{m}$, we conclude that the maximum intensity occurs at $P_{a}=1.46 \mathrm{~atm}$. The number of emitted photons at this point is $\approx 8 \times 10^{6}$, which is in close agreement with the reported value in Refs. 2, 38 for well-cooled water. Following the diffusive equilibria with varying $P_{a}$, we obtain the photon numbers displayed in Fig. 6 in a $P_{a}$ interval, which is clearly inside the range reported for SBSL experiments. The weakest light pulses (at high ambient temperatures) are reported to have $\sim 10^{4}$ photons; according to our model, these correspond to bubbles of size $R_{0} \approx 3.5 \mu \mathrm{m}$.

In Ref. 39 of case (ii), pulse widths as a function of $P_{a}$ were given, reproduced in Fig. 7(a). The corresponding calculations in the present model yield the values in Fig. 7(b) as a function of $P_{a}$. The shortest pulse widths are obtained for the smallest bubbles at the turning point of the diffusive equilibrium line (cf. Fig. 5). The range of pulse widths agrees well with experiment; also, the shape of the growth with $P_{a}$ is reproduced, apart from the apparent saturation in FWHM at the lowest gas concentrations and the highest $P_{a}$ [circles in Fig. 7(a)]. These particular experimental data, however, display a quite large uncertainty in the measurement of concentration; for the theoretical curve in Fig. 7(b) a mean concentration was used. The absolute values of $P_{a}$ deviate from the experiment by about $0.10-0.15 \mathrm{~atm}$. Apart from the obvious inaccuracies and simplifying assumptions in our model, this could also, in part, result from the experimental difficulty of measuring the true driving amplitude $P_{a}$ (a systematic error of about the size of the reported deviations is possible ${ }^{59}$ ).

The data in (iii) do not have calibrated $P_{a}$ values, so that a direct comparison is not possible. Nevertheless, the observed pulse widths again agree with the range of values computed from the presented model. The important observation of longer widths for the longer-wavelength part of the spectrum when working at low $T_{\infty}$ was already explained in Sec. IV.

The most extensive dataset is presented in case (iv), ${ }^{41}$ for $\mathrm{Ar}, \mathrm{Xe}$, and $\mathrm{He}$ at $0.004 \mathrm{~atm}$ (3 Torr) partial pressure $\left(c_{\infty} / c_{0} \approx 0.4 \%\right)$. In that work, the intensities are given relative to the maximum intensity obtained from a $0.2 \mathrm{~atm}(150$ Torr) air bubble (i.e., a $0.002 \mathrm{~atm}$ or 1.5 Torr Ar bubble), which is not given in absolute numbers for this case of $f$ $=34 \mathrm{kHz}$ driving. Figure 1 of the experimental paper ${ }^{41}$ shows examples of the pulse widths in air at that partial pressure, together with the fraction of maximum intensity. As we can compute the intensity corresponding to the given pulse widths, we can deduce the maximum intensity for 0.2 atm air from this data. In Fig. 8 we have normalized all theoretical intensity data by this value $\left(\approx 6.5 \times 10^{5}\right.$ photons or $3.9 \times 10^{-13} \mathrm{~J}$ emitted per oscillation cycle).

One of the most striking features of Fig. 2(a) in Ref. 41 is the high intensity of xenon SBSL, which is clearly reproduced in our Fig. 8(a). The values for both pulse width and relative intensity are in very good accord with the experiment. Even more important is the fact that the dependence of the pulse width on the intensity agrees very well with the measurements, because this indicates that the basic mechanisms of light emission are represented correctly in the present approach. Figure 8(b) corresponds to Fig. 2(b) of Ref. 41, showing the same graph in the region of smaller intensities for Xe and Ar bubbles at $c_{\infty} / c_{0}=0.4 \%$, as well as for $\mathrm{Ar}$ at $c_{\infty} / c_{0}=0.03 \%$, corresponding to the $0.026 \mathrm{~atm}$ (20 Torr) air bubbles used in the experiment. The dependences of the pulse widths on the intensities again compare well with the measured values, especially considering the simplicity of the model we presented. Moreover, at the same gas concentration, Xe and Ar bubbles follow practically identical curves in this diagram, although a given pulse width corre-
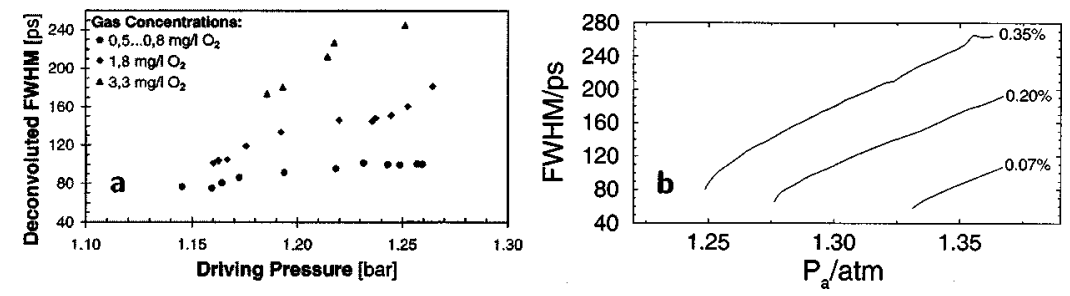

FIG. 7. (a) Measured pulse widths, reproduced from Ref. 39 for argon bubbles driven at $f=20 \mathrm{kHz}$ at three different concentrations of dissolved air (the oxygen concentrations are given here). (b) Calculated pulse widths for diffusively stable argon bubbles at relative gas concentrations corresponding to the experimental values in (a). The stable $R_{0}$ values can be read off Fig. 5. The discrepancy on the $P_{a}$ axis to experiment may be due to inaccuracies of the model or to the difficulties of calibrating $P_{a}$ in the experiment. The pulse widths and the shape of their $P_{a}$ dependence are, however, in good agreement, with the exception of the apparent saturation of the experimental data at the lowest concentrations and the highest $P_{a}$. 

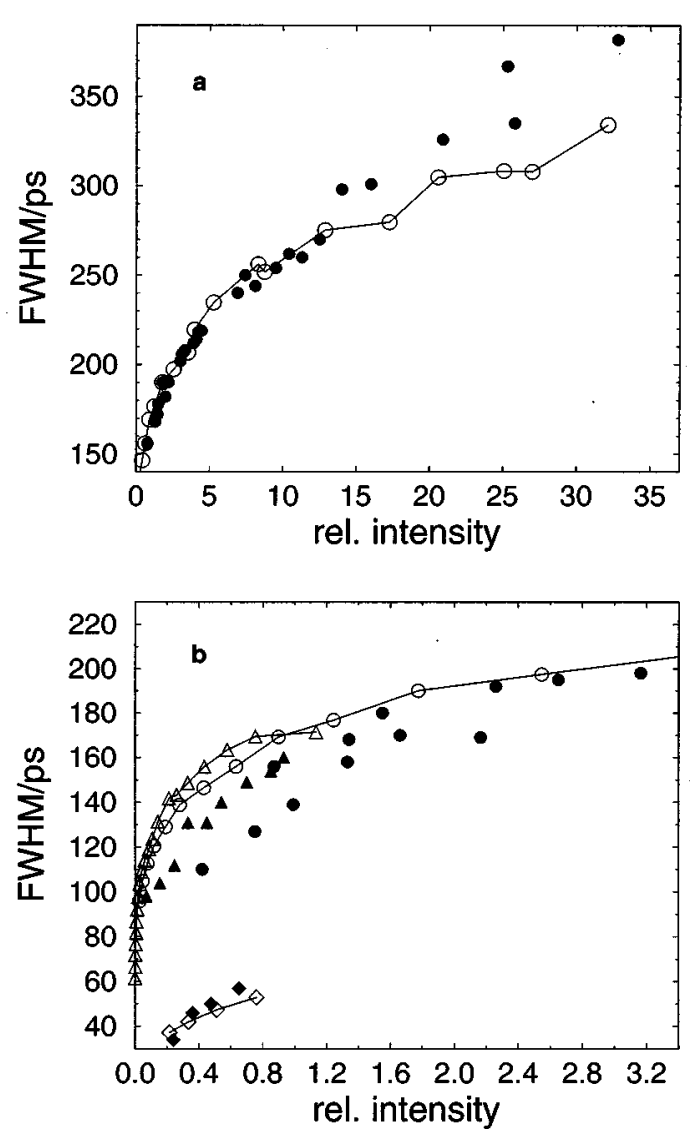

FIG. 8. (a) Calculated pulse widths (open circles) for diffusively stable xenon bubbles at $f=34 \mathrm{kHz}$ and $c_{\infty} / c_{0}=0.4 \%$ (as reported in Ref. 41 ) as a function of pulse intensity. The filled symbols are the experimental values of Ref. 41 . The intensities are given relative to the maximum intensity for 0.2 atm (150 Torr) air (argon concentration $c_{\infty} / c_{0} \approx 0.2 \%$ ). In experiment the brightest bubbles were obtained at lower water temperatures, where stronger forcing and larger bubble radii are possible (Ref. 16). (b) A comparison of the pulse widths for diffusively stable Ar (triangles) and Xe (circles) bubbles for the same parameters as in (a), now in the range of smaller intensities and widths. Solid symbols denote experimental data, open symbols theoretical results. Diamonds represent values for argon at $c_{\infty} / c_{0}=0.03 \%$, corresponding to the 0.026 atm (20 Torr) air experiment reported in Ref. 41.

sponds to different $P_{a}$ and $R_{0}$ for $\mathrm{Ar}$ and Xe, respectively. This is another experimental feature of Fig. 2(b) in Ref. 41 reproduced within the present approach. Note that the largest numerical values for pulse widths and intensities displayed for Ar and $\mathrm{Xe}$ at $c_{\infty} / c_{0}=0.4 \%$ in Figs. 8(a) and 8(b) occur for bubbles of the same size and at the same driving pressure: in both cases, $P_{a}^{\max } \approx 1.47 \mathrm{~atm}$ and $R_{0}^{\max } \approx 5.5 \mu \mathrm{m}$. This is consistent with the concept of shape instabilities as the limiting factor for $R_{0}$ and $P_{a}$, cutting off the growth along the curve of diffusive equilibria (cf. Fig. 5), as the shape instability thresholds are virtually identical for both $\mathrm{Ar}$ and Xe.

Note that the dataset for 0.026 atm air [diamonds in Fig. 8(b)] probes all salient features of the theory presented here: The values for $P_{a}$ and $R_{0}$ are calculated using the diffusive stability formalism, under the assumption that only argon remains in the bubble (the relative concentration is thus taken to be $0.03 \%$, not $3 \%$ ). From the resulting parameters of bubble dynamics, the light emission is calculated. The con- siderable change in gas concentration from $0.4 \%$ to $0.03 \%$ in the experiments results in a corresponding change in the functional dependence of width on intensity, which is very well covered by the present calculations; see Fig. 8(b).

The values for helium from Ref. 41 could not be reproduced; the computations yield much too faint bubbles if only the material parameters are changed, mostly because of the huge ionization energy $E_{\text {ion }}(\mathrm{He}) \approx 24.6 \mathrm{eV}$. While experiments report that He bubbles are about a factor of 10 less bright $^{2}$ than xenon, the present model would predict about four orders of magnitude difference. A possible explanation is our neglect of the presence of water vapor molecules orafter the bubble becomes hot enough-the radicals derived from them. They are also capable of light absorption and emission, probably at lower $T$ than the noble gases, because their ionization energies are lower ${ }^{4}$ (in the range of $\approx 14 \mathrm{eV}$ for both $\mathrm{H}$ and $\mathrm{O}$ ). Thus, for $\mathrm{He}$ with its extremely high ionization energy, it is possible that it is rather the "impurities" (water vapor) in the gas that are responsible for the major part of the light emission than the noble gas itself (Ref. 57; cf. also Ref. 58).

\section{SUMMARY, PREDICTIONS, AND CONCLUSIONS}

In summarizing, we repeat that the main contributors to the light emission from the hot bubble are thermal bremsstrahlung and recombination radiation from an ensemble of atoms, ions, and electrons. All these processes depend crucially on the degree of ionization, which is generally quite small ( $\sim 1 \%$ for $\mathrm{Ar}, \lessgtr 10 \%$ for $\mathrm{Xe}$ ). The inherent blackbody character of the thermal radiation is changed because of the small opacity or absorptivity of the bubble. Its small optical thickness leads to a considerable reduction of the total emitted intensity compared with the results for a blackbody of infinite opacity, thus coming much closer to the observed intensities. Moreover, the transparency of the bubble accounts for the experimentally observed short duration and approximate wavelength independence of the pulse widths, which calculations assuming an ideal blackbody fail to explain. When using explicit formulas for the opacity contributions due to absorption and reemission of photons in the gas bubble (Secs. IV and V), the simple approach presented here does surprisingly well in predicting the pulse shapes and widths, as well as the spectral shape and intensity of the light emission for both Ar and Xe SBSL bubbles.

It is the various features of the optical thickness $\tau_{\lambda}(t)$ $=2 \kappa_{\lambda}[T(t)] R(t)$ that are responsible for the characteristic deviations of the emitted light pulses from those of simple black body theory. In particular, the optical thickness is proportional to an exponential switch that determines the pulse widths, namely,

$$
\tau_{\lambda}(t) \propto \exp \left(-\frac{E_{\text {ion }}}{k_{\mathrm{B}} T(t)}\right) .
$$

Thus, a characteristic energy scale-the ionization energy of the noble gas atom-enters the picture, which is not present in blackbody radiation. Even the maximum temperatures at collapse are still fairly small compared to $E_{\text {ion }} / k_{\mathrm{B}}$ for noble gases, making the switch extremely sensitive. This explains 
both the reduced width and the reduced intensity of the pulses in the optically thin case. As the switch (26) is $\lambda$ independent, the counterintuitive finding of nearly equal pulse widths in the red and UV part of the spectrum is a natural consequence of the model. The absolute value of photon absorption, however, is $\lambda$ dependent and leads to a pronouncedly larger opacity for longer wavelengths due to the terms proportional to $\lambda^{2}$ and $\lambda^{3}$ in (23) and (25), respectively [cf. Fig. 3(c)].

Note that for an only slightly different range of values for $E_{\text {ion }} / k_{\mathrm{B}} T$, the computation would have resulted in bubbles that are either very opaque and would behave similar to blackbody bubbles (see Sec. III), or they would be optically very thin (transparent) all of the time, with emission rates many orders of magnitude lower than those observed experimentally. This strong sensitivity to $E_{\text {ion }}$ gives us further confidence that our interpretation captures the essentials of the light emission mechanism of sonoluminescing bubbles.

The most reassuring feature is probably the agreement with the experimentally observed dependence of the pulse width on the pulse intensity (Fig. 8), two accurately measurable physical quantities for which unambiguous experimental data are available. The successful reproduction of this relationship over the whole range of observed intensities and pulse widths is a strong indication that the present theory is indeed generally applicable for SBSL bubbles and not just in special cases.

According to the now widely accepted picture of molecule dissociation in hot SBSL bubbles, ${ }^{14}$ the interior of stably oscillating SBSL bubbles prepared with some gas mixture eventually consists of almost pure noble gases, whose first ionization potentials vary from $24.6(\mathrm{He})$ to $12.1 \mathrm{eV}$ $(\mathrm{Xe})$. As the quotient $E_{\text {ion }} / k_{\mathrm{B}} T$ becomes smaller for Xe, and the temperatures become higher because of the smaller thermal diffusivity $\chi$ of Xe computed from (8), the switch (26) loses its sensitivity. Consequently, Xe bubbles should, according to the present model (i) be brighter, as seen in Fig. 8, and (ii) experience a less rapid cutoff of light emission due to increased opacity. Therefore, not only should the pulse width for Xe be longer, but we predict that also its variation with wavelength should be detectable for the brightest bubbles, which have pulse widths of more than 300 ps; see Fig. 4(b).

Quite generally, a $\lambda$ dependence of the pulse width will be more pronounced for more violent collapses ("upscaled" sonoluminescence), which yield longer pulse widths as well as higher temperatures, intensities, and opacities. One possibility of upscaling is lowering the ambient water temperature $T_{\infty}$, which indeed leads to the expected detection of the pulse width variation, even for argon. ${ }^{42}$ Also, lower driving frequencies $f$ should act in the same direction, because bubbles exhibit stronger collapses at smaller frequencies. ${ }^{17,37}$ While we expect the light emission to be much stronger at lower $f,{ }^{37}$ the effects on the maximum bubble temperature will be less pronounced. A rather pronounced change of the experimental parameters (while maintaining stable bubbles) seems to be necessary to raise $T_{\max }$ considerably; in all cases calculated above, the maximum bubble temperatures lie in a relatively narrow window of $\sim 20000-25000 \mathrm{~K}$ (much smaller than $E_{\text {ion }} / k_{\mathrm{B}}$, even for $\mathrm{Xe}$ ).

Our aim in this work is to demonstrate the feasibility of calculating realistic light emission for SBSL using very simple physics. The dynamical equations contain just the Rayleigh-Plesset equation, a rudimentary modeling of heat exchange between the gas bubbles and the ambient water via a polytropic exponent, and thermal photon emission, taking proper care of the reduced gas opacity. The emission mechanisms mentioned above, as well as all other elements of the theory, are well-known physical effects and no exotic "new physics" has to be proposed. In fact, there is a growing consensus among theorists that SBSL light can be accounted for by photon emission involving the relatively few ions and free electrons present in the interior of the collapsed bubble. This includes the computations by Moss et al. ${ }^{4}$ and Yasui, ${ }^{25}$ the CEM model, ${ }^{6}$ and bremsstrahlung calculations. ${ }^{26}$ Experimental findings have paralleled this convergence, as all data on the pulse widths and their dependence on parameters agree very well today. We are confident that the essentials, as well as the details of SBSL emission, can be understood along these lines. As the principle of Occam's razor tells us, we should not choose a more complicated model if a simple explanation already agrees with reality.

In the present paper, the light emission of SBSL bubbles is treated as an integral part of the bubble dynamical calculations governed by the Rayleigh-Plesset equation (1). All the various features of the RP-SL bubble approach-shape stability, diffusive stability, chemical processes in the bubble, and, finally, light emission as thermal emission from an optically thin body-fit together and give a consistent picture of SBSL in its whole experimental parameter range. There are no free parameters in the calculations; no functions or constants are chosen arbitrarily. Due to the simplicity of the approach, the complete parameter space of SL is accessible to these calculations. With the predictions given above, measurements will be able to show if this approach holds true, whose most satisfactory feature may be its ability to explain as outlandish an effect as single bubble sonoluminescence by the application of very simple physical principles.

\section{ACKNOWLEDGMENTS}

We thank Stephan Koehler, Willy Moss, and Howard Stone for valuable discussions, Bruno Gompf and Wolfgang Eisenmenger for allowing us to reproduce their experimental Fig. 7(a), and Peter Hänggi for stimulating questions. Financial support through the DFG Grants No. SFB 185/D8 and No. Lo556/3 is gratefully acknowledged.

${ }^{1}$ D. F. Gaitan, "An experimental investigation of acoustic cavitation in gaseous liquids," Ph.D. thesis, The University of Mississippi, 1990; D. F. Gaitan, L. A. Crum, R. A. Roy, and C. C. Church, "Sonoluminescence and bubble dynamics for a single, stable, cavitation bubble," J. Acoust. Soc. Am. 91, 3166 (1992).

${ }^{2}$ B. P. Barber, R. A. Hiller, R. Löfstedt, S. J. Putterman, and K. R. Weninger, "Defining the unknowns of sonoluminescence," Phys. Rep. 281, 65 (1997).

${ }^{3}$ C. C. Wu and P. H. Roberts, "Shock-wave propagation in a sonoluminescing gas bubble," Phys. Rev. Lett. 70, 3424 (1993); “A model of sonoluminescence,'” Proc. R. Soc. London, Ser. A 445, 323 (1994). 
${ }^{4}$ W. Moss, D. Clarke, and D. Young, "Calculated pulse widths and spectra of a single sonoluminescing bubble,"' Science 276, 1398 (1997).

${ }^{5}$ L. Frommhold and A. A. Atchley, "Is sonoluminescence due to collisioninduced emission?,' Phys. Rev. Lett. 73, 2883 (1994).

${ }^{6}$ L. S. Bernstein and M. R. Zakin, "Confined electron model for singlebubble sonoluminescence," J. Phys. Chem. 99, 14619 (1995).

${ }^{7}$ A. Prosperetti, "A new mechanism for sonoluminescence," J. Acoust. Soc. Am. 101, 2003 (1997).

${ }^{8} \mathrm{~K}$. Yasui, "Effects of thermal conduction on bubble dynamics near the sonoluminescence threshold,' J. Acoust. Soc. Am. 98, 2772 (1995); “Alternative model of single-bubble sonoluminescence," Phys. Rev. E 56, 6750 (1997).

${ }^{9}$ V. Q. Vuong and A. J. Szeri, "Sonoluminescence and diffusive transport," Phys. Fluids 8, 2354 (1996).

${ }^{10}$ M.-C. Chu and D. Leung, "Effects of thermal conduction in sonoluminescence," J. Phys.: Condens. Matter 9, 3387 (1997).

${ }^{11}$ L. Yuan, H. Y. Cheng, M. C. Chu, and P. T. Leung, "Physical parameters affecting sonoluminescence: A self-consistent hydrodynamic study," Phys. Rev. E 57, 4265 (1998).

${ }^{12}$ M. Brenner, D. Lohse, and T. Dupont, "Bubble shape oscillations and the onset of sonoluminescence,"' Phys. Rev. Lett. 75, 954 (1995); see also the reply to the comment by S. J. Putterman and P. H. Roberts on the same paper, ibid. 80, 3668 (1998).

${ }^{13}$ S. Hilgenfeldt, D. Lohse, and M. P. Brenner, "Phase diagrams for sonoluminescing bubbles," Phys. Fluids 8, 2808 (1996).

${ }^{14}$ D. Lohse, M. P. Brenner, T. Dupont, S. Hilgenfeldt, and B. Johnston, "Sonoluminescing air bubbles rectify argon," Phys. Rev. Lett. 78, 1359 (1997); D. Lohse and S. Hilgenfeldt, "Inert gas accumulation in sonoluminescing bubbles," J. Chem. Phys. 107, 6986 (1997).

${ }^{15}$ M. P. Brenner, S. Hilgenfeldt, and D. Lohse, "The hydrodynamical/ chemical approach to sonoluminescence," in Sonochemistry and Sonoluminescence, NATO-ASI series C, edited by L. Crum, T. Mason, J. Reisse, and K. Suslick (Kluwer, Dordrecht, 1999), Vol. 524.

${ }^{16}$ S. Hilgenfeldt, D. Lohse, and W. Moss, "Water temperature dependence of single bubble sonoluminescence," Phys. Rev. Lett. 80, 1332 (1998).

${ }^{17}$ S. Hilgenfeldt, M. P. Brenner, S. Grossmann, and D. Lohse, "Analysis of Rayleigh-Plesset dynamics for sonoluminescing bubbles,' J. Fluid Mech. 365, 171 (1998)

${ }^{18}$ L. Kondic, C. Yuan, and C. K. Chan, "Ambient pressure and singlebubble sonoluminescence," Phys. Rev. E 57, R32 (1998).

${ }^{19}$ A. Prosperetti and Y. Hao, "Modeling of spherical gas bubble oscillations," Philos. Trans. R. Soc. London (1998).

${ }^{20}$ D. Lohse, M. P. Brenner, and S. Hilgenfeldt, "Phase diagrams for sonoluminescing bubbles,'” J. Acoust. Soc. Am. 100, 2678 (1996).

${ }^{21}$ F. Lepoint-Mullie, T. Lepoint-Mullie, and A. Henglein, "Single-bubble sonochemistry,', in Ref. 15.

${ }^{22}$ G. Holt and F. Gaitan, "Observation of stability boundaries in the parameter space of single bubble sonoluminescence,'” Phys. Rev. Lett. 77, 3791 (1996).

${ }^{23}$ F. Gaitan and G. Holt, "Nonlinear bubble dynamics and light emission in single-bubble sonoluminescence,"' J. Acoust. Soc. Am. 103, 3046 (1998).

${ }^{24}$ R. E. Apfel and J. A. Ketterling, "Sonoluminescence stability for gas pressures down to 0.01 Torr," J. Acoust. Soc. Am. 103, 3076 (1998).

${ }^{25} \mathrm{~K}$. Yasui, "Mechanism of single bubble sonoluminescence" (preprint).

${ }^{26}$ L. Frommhold, "Electron-atom bremsstrahlung and the sonoluminescence of rare gas bubbles," Phys. Rev. E 58, 1899 (1998).

${ }^{27} \mathrm{~L}$. Rayleigh, "On the pressure developed in a liquid on the collapse of a spherical bubble," Philos. Mag. 34, 94 (1917).

${ }^{28}$ M. S. Plesset, "The dynamics of cavitation bubbles," J. Appl. Mech. 16, 277 (1949)

${ }^{29}$ C. E. Brennen, Cavitation and Bubble Dynamics (Oxford University Press, Oxford, 1995).

${ }^{30}$ R. Löfstedt, B. P. Barber, and S. J. Putterman, "Toward a hydrodynamic theory of sonoluminescence," Phys. Fluids A 5, 2911 (1993).

${ }^{31}$ D. R. Lide, Handbook of Chemistry and Physics (CRC Press, Boca Raton, 1991).
${ }^{32}$ A. Prosperetti, "Thermal effects and damping mechanisms in the forced radial oscillations of gas bubbles in liquids," J. Acoust. Soc. Am. 61, 17 (1977).

${ }^{33}$ M. Plesset and A. Prosperetti, "Bubble dynamics and cavitation," Annu. Rev. Fluid Mech. 9, 145 (1977).

${ }^{34}$ R. Löfstedt, K. Weninger, S. J. Putterman, and B. P. Barber, "Sonoluminescing bubbles and mass diffusion," Phys. Rev. E 51, 4400 (1995).

${ }^{35}$ R. A. Hiller, K. R. Weninger, S. J. Putterman, and B. P. Barber, "Effect of noble gas doping in single-bubble sonoluminescence," Science 266, 248 (1994).

${ }^{36}$ T. J. Matula and L. A. Crum, "Evidence for gas exchange in singlebubble sonoluminescence," Phys. Rev. Lett. 80, 865 (1998).

${ }^{37}$ S. Hilgenfeldt and D. Lohse, "Predictions for upscaling sonoluminescence," Phys. Rev. Lett. 82, 1036 (1999).

${ }^{38}$ B. P. Barber, C. C. Wu, R. Löfstedt, P. H. Roberts, and S. J. Putterman, "Sensitivity of sonoluminescence to experimental parameters," Phys. Rev. Lett. 72, 1380 (1994).

${ }^{39}$ B. Gompf, R. Günther, G. Nick, R. Pecha, and W. Eisenmenger, "Resolving sonoluminescence pulse width with time-correlated single photon counting,"' Phys. Rev. Lett. 79, 1405 (1997).

${ }^{40}$ R. Pecha, B. Gompf, G. Nick, and W. Eisenmenger, "Resolving the sonoluminescence pulse shape with a streak camera," Phys. Rev. Lett. 81, 717 (1998).

${ }^{41}$ R. A. Hiller, S. J. Putterman, and K. R. Weninger, "Time-resolved spectra of sonoluminescence,' Phys. Rev. Lett. 80, 1090 (1998).

${ }^{42}$ M. J. Moran and D. Sweider, "Measurements of sonoluminescence temporal pulse shape,'” Phys. Rev. Lett. 80, 4987 (1998).

${ }^{43}$ A. Prosperetti, "Bubble phenomena in sound fields," Part I and II, Ultrasonics 22, 69 and 115 (1984).

${ }^{44} \mathrm{~K}$. Weninger, B. Barber, and S. Putterman, "Pulsed Mie scattering measurements of the collapse of a sonoluminescing bubble,' Phys. Rev. Lett. 78, 1799 (1997).

${ }^{45}$ G. A. Delgadino and F. J. Bonetto, "Velocity interferometry technique used to measure the expansion and compression phases of a sonoluminescent bubble,"' Phys. Rev. E 56, R6248 (1997).

${ }^{46}$ J. O. Hirschfelder, C. F. Curtiss, and R. B. Bird, Molecular Theory of Gases and Liquids (Wiley, New York, 1954).

${ }^{47}$ H. S. Carslaw and J. C. Jaeger, Conduction of Heat in Solids (Clarendon, Oxford, 1989).

${ }^{48}$ R. Hiller, S. J. Putterman, and B. P. Barber, "Spectrum of synchronous picosecond sonoluminescence," Phys. Rev. Lett. 69, 1182 (1992).

${ }^{49}$ Y. B. Zel'dovich and Y. P. Raizer, Physics of Shock Waves and HighTemperature Hydrodynamic Phenomena (Academic, New York, 1966), Vols. I and II.

${ }^{50}$ R. Siegel and J. R. Howell, Thermal Radiation Heat Transfer (McGrawHill, New York, 1972).

${ }^{51}$ V. L. Ginzburg, Propagation of Electromagnetic Waves in Plasma (Gordon and Breach, New York, 1962).

${ }^{52}$ S. C. Brown, Basic Data of Plasma Physics (MIT Press, Cambridge, 1966).

${ }^{53}$ R. F. Bacher and S. Goudsmit, Atomic Energy States (McGraw-Hill, New York, 1932).

${ }^{54}$ D. F. Gaitan, A. A. Atchley, S. D. Lewia, J. T. Carlson, X. K. Maruyama, M. Moran, and D. Sweider, "Spectra of single-bubble sonoluminescence in water and glycerin-water mixtures," Phys. Rev. E 54, 525 (1996).

${ }^{55}$ L. S. Bernstein, M. R. Zakin, E. B. Flint, and K. S. Suslick, "Thermometry using molecular and continuum sonoluminescence,' J. Phys. Chem. 100, 6612 (1996); L. S. Bernstein, "An interpretation of the MBSL temperatures deduced from metal atom emission," J. Acoust. Soc. Am. 103, 2924 (1998).

${ }^{56}$ D. Mihalas, Stellar Atmospheres (Freeman, San Francisco, 1978).

${ }^{57}$ W. Moss (private communication, 1998).

${ }^{58}$ W. C. Moss and D. A. Young, "Computed spectral and temporal emissions from a sonoluminescing bubble,' J. Acoust. Soc. Am. 103, 3076 (1998).

${ }^{59} \mathrm{~B}$. Gompf (private communication, 1998). 